Meta

Journal des traducteurs

Translators' Journal

\title{
Lexicomatique, compréhensionnisation et extensionnisation
}

\section{René Tondji-Simen}

Volume 42, numéro 2, juin 1997

Lexicologie et terminologie II (1) et Traduction et post-colonialisme en Inde

Translation and Postcolonialism: India (2)

URI : https://id.erudit.org/iderudit/004145ar

DOI : https://doi.org/10.7202/004145ar

Aller au sommaire du numéro

Éditeur(s)

Les Presses de l'Université de Montréal

ISSN

0026-0452 (imprimé)

1492-1421 (numérique)

Découvrir la revue

Citer cet article

Tondji-Simen, R. (1997). Lexicomatique, compréhensionnisation et extensionnisation. Meta, 42(2), 364-373. https://doi.org/10.7202/004145ar

\section{Résumé de l'article}

Cet article propose des pistes de solution aux problèmes de décomposage du réel que posent les phénomènes d'extension et de compréhension de termes en lexicographie bilingue. 


\title{
LEXICOMATIQUE, COMPRÉHENSIONNISATION ET EXTENSIONNISATION*
}

RENÉ TONDJI-SIMEN

GRESLET, Université de Montréal, Montréal. Canada

\begin{abstract}
Résumé
Cet article propose des pistes de solution aux problèmes de décomposage du réel que posent les phénomènes d'extension et de compréhension de termes en lexicographie bilingue.
\end{abstract}

\section{Abstract}

This articles proposes a framework for dealing with problems relating to comprehension and extension of meaning in bilingual lexicography.

\section{INTRODUCTION}

La «compréhensionnisation» et l'«extensionnisation» posent à la traduction, et sûrement à la traductique et encore plus à la lexicomatique, des problèmes de gestion informatisée d'équivalence de type diatopique, spécifique/générique, partie/tout et topolectal, selon qu'on part de la langue $\mathrm{A}$ ou de la langue B.

La lexicographie bilingue traditionnelle se heurte au sempiternel problème du découpage du réel d'une culture à l'autre, d'une langue à l'autre. Les produits lexicographiques, beaucoup plus sur support papier actuellement, sont statiques et ne permettent pas une bonne communication entre les cultures et les langues. Ceux qui sont en chantier seront appelés à être dynamiques, car ils devront être disponibles sur réseaux et autoroute électroniques. Quelle approche pouvons-nous préconiser ou privilégier pour solutionner ces problèmes, ou du moins pour nous préparer à livrer du bon produit lexicographique à cet univers électronique qui s'ouvre et dans lequel les gens, parce que plus exigeants, voudront avoir les équivalents appropriés au bout des doigts? Lexicomatique, oui! Mais que devrat-elle faire pour répondre?

\section{DÉFINITIONS}

\section{a) Lexicomatique}

La lexicomatique est synonyme de la lexicologie informatique et correspond à l'ensemble des méthodes, des techniques et des pratiques utilisant l'informatique dans l'étude et l'exploitation des corpus textuels.

\section{b) Compréhensionnisation et extensionnisation}

Quand, pour la première fois, Port-Royal employa les expressions compréhension et extension dans son ouvrage de grammaire, ce fut pour distinguer les significations d'une idée. Par la suite, on les utilisa également pour différencier les acceptions d'un terme. En linguistique et en logique, ces expressions sont devenues synonymes technolectaux de connotation et de dénotation.

Pour Duval (1991: 2820), la compréhension d'un concept regroupe l'ensemble des caractères spécifiques de ce concept. Alors que Arnauld et Nicole appellent compréhension 
d'une idée les attributs que cette idée «enferme en soi, et qu'on ne peut lui ôter sans la détruire, comme la compréhension de l'idée du triangle enferme extension, figure, trois lignes, trois angles, et l'égalité de ces trois angles à deux droits, etc.» (Arnauld et Nicole 1878: 72).

L'extension d'un concept regroupe, selon Alain Duval (1991 : 2820), l'ensemble des objets auxquels ce concept s'applique. Antoine Arnauld et Pierre Nicole, quant à eux, appellent extension d'une idée «les sujets à qui cette idée convient; ce qu'on appelle aussi les inférieurs d'un terme général, qui, à leur égard, est appelé supérieur, comme l'idée du triangle en général s'étend à toutes les diverses espèces de triangles» (Arnauld et Nicole 1878: 72).

\section{c) Équivalence}

Nous pouvons définir l'équivalence comme la relation qui met en rapport de synonymie des dénominations de langues différentes représentant la même notion. Généralement, quand on traite deux dénominations d'équivalentes, on sous-entend qu'elles sont interchangeables.

Cependant, pour que deux dénominations soient interchangeables, il faudrait que la notion à laquelle elles renvoient dans les deux langues soit identique aussi bien en compréhension qu'en extension. Or, l'équivalence se présentant en terme de degré, on a trois types principaux d'équivalence : $a$ ) équivalence totale (parfaite, absolue ou stricte), b) équivalence relative, $c$ ) équivalence partielle.

- L'équivalence totale (parfaite, absolue ou stricte) est celle qui met en relation deux termes dont le découpage notionnel dans les deux langues auxquelles ils appartiennent est le même. Dans ce cas, le découpage de la réalité coïncide, et la correspondance entre le terme et la notion est biunivoque, isomorphique. Le terme de la langue $\mathrm{A}$ est à tout point de vue équivalent au terme de la langue $B$.

- L'équivalence relative: seul le niveau de langue différencie le terme de la langue A de celui de la langue $B$.

- L'équivalence partielle : le terme de la langue A présente une extension ou une compréhension différente de celle que sous-tend le terme de la langue B. Chacune des langues découpe la réalité à sa façon propre. Les structures sémantiques des deux langues considérées sont différentes. L'équivalence partielle comprend les équivalences que Goffin (1985: 23-25) appelle absolue $1: 2$, à extension différente, allomorphique.

L'équivalence partielle nous intéresse particulièrement, et elle constitue la base de notre étude.

\section{3. ÉQUIVALENCE PARTIELLE}

\section{a) Le découpage du réel}

Des déterminismes linguistiques, historiques, socioculturels et géographiques, entre autres, pèsent sur les configurations notionnelles des expressions que nous employons. D'une langue à l'autre, ils influencent le degré de correspondance des notions et la valeur sémantique des unités linguistiques qui leur sont associées. Comme le dit Goffin (1985: 18-19):

Au sein du domaine confus de l'environnement non linguistique, la langue opère au moyen de signes des découpages différents d'une langue à l'autre [...] Nous percevons la réalité de telle ou telle manière selon les catégories de la pensée que nous impose notre langue [...] Décrivant un phénomène, le savant ne décrit jamais qu'un élément arbitrairement isolé de l'univers et procède lui aussi à des découpages que la nature ignore et qui sont forcément sa création. Il segmente des continuums. Lorsque l'on compare la terminologie d'un même domaine en plusieurs langues, on constate la complexité des points de vue et la multiplicité des «visions» d'un même phénomène, chaque observation engendrant le phénomène. 
En bref, loin d'être arbitraire, le découpage du réel obéit à une logique différente d'une langue à l'autre, d'un individu ou d'une communauté à l'autre. Nous ajouterions que le réel, lui aussi, se présente différemment d'un milieu à l'autre (qu'il s'agisse du milieu social, culturel, géographique ou autre). Ces différences donnent lieu à de nombreux problèmes d'équivalence imputables surtout aux différences de compréhension et d'extension des termes considérés. Une notion a une seule dénomination dans la langue A alors que dans la langue $B$, il faut deux ou plusieurs dénominations pour couvrir la même notion. L'extension et la compréhension des termes posent par conséquent des problèmes particuliers d'une langue, d'un milieu ou d'un domaine à l'autre. Les termes de couleur, de parenté, de liberté, des droits de la personne ne sont que quelques-uns parmi tant d'autres dont les notions s'élargissent ou se rétrécissent selon les sociétés dans lesquelles on se trouve.

Il en est ainsi de certaines expressions françaises et allemandes que Goffin (1985: 19) a analysées. La structuration sémantique de ces expressions présente des différences considérables en français et en allemand. Nous en faisons la récapitulation dans le tableau ci-après. Le terme français (dans la colonne de gauche) couvre une notion; la même notion est couverte en allemand par deux termes, chacun de ces derniers ne couvrant qu'une partie de la notion.

\begin{tabular}{|l|l|}
\hline \multicolumn{1}{|c|}{ TERME FRANÇAIS } & \multicolumn{1}{c|}{ ÉQUIVALENTS ALLEMANDS } \\
\hline \multirow{2}{*}{$\begin{array}{l}\text { durée de mise en température } \\
\text { (ou durée de chauffage) }\end{array}$} & Anwärmdauer \\
\cline { 2 - 2 } & Durchwärmdauer \\
\hline \multirow{2}{*}{ surchauffe } & Überhitzen \\
\cline { 2 - 2 } & Überzeihzen \\
\hline \multirow{2}{*}{ déformation de trempe } & Verziehen \\
\cline { 2 - 2 } & Massänderung \\
\hline \multirow{2}{*}{ décarburation } & Auskohlung \\
\cline { 2 - 2 } & Abkohlung \\
\hline
\end{tabular}

Dans le cadre du français et de l'anglais, la situation n'est pas différente. Les expressions traffic warden, lollipop man / lady et meter maid que nous empruntons à Duval (1991: 2820) révèlent des problèmes de compréhension et d'extension fort intéressants entre ces deux langues.

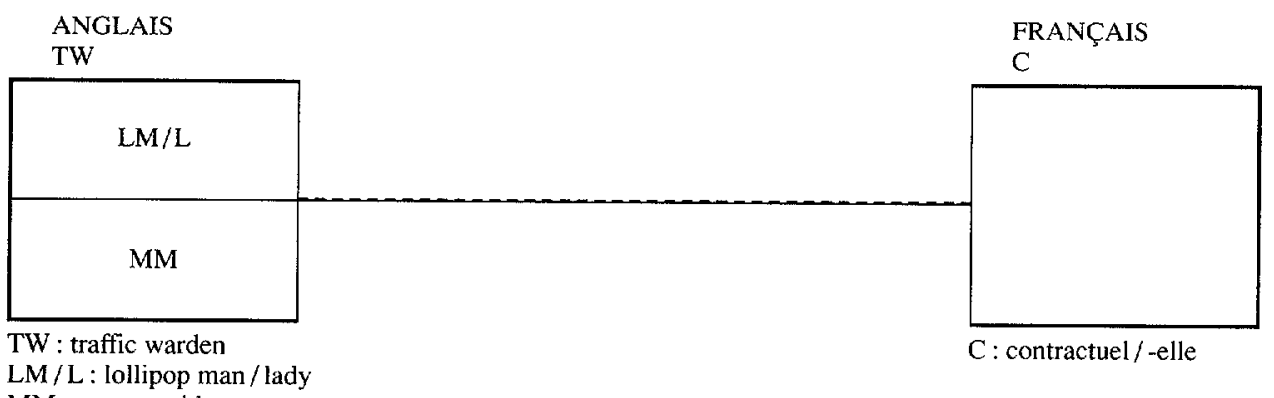

$\mathrm{MM}:$ meter maid 
Le terme anglais traffic warden et le terme français contractuel/-elle entretiennent une relation d'équivalence totale. Ils renvoient à une même notion, cette notion ayant la même extension et la même compréhension en français et en anglais, à savoir : auxiliaires de police, généralement des femmes ou des retraités, chargés de s'occuper des problèmes de circulation dans les villes (Duval 1991 : 2820).

Cependant, en anglais, traffic warden a deux synonymes partiels: lollipop man/lady et meter maid. Comme illustré dans la figure ci-dessus, chacun d'eux ne recouvre qu'une partie du champ notionnel de traffic warden. Ils ne sont donc pas synonymes l'un et l'autre. Chacun d'eux a sa compréhension (ses caractères spécifiques). Cela étant, ils sont des synonymes spécifiques du terme générique traffic warden.

Pour ce qui est de l'équivalent français de ces trois termes anglais, aucune difficulté apparente ne se présente. Le terme français contractuel/-elle est l'équivalent de tous les trois. Mais attention! En réalité, les appariements lollipop man/lady - contractuel/-elle et meter maid - contractuel/-elle sont trompeurs.

\section{b) L'importance du référent}

Dans toute communication (monolingue ou bilingue), le référent est très important, sinon essentiel. Pour bien illustrer le problème, nous construisons trois triangles.
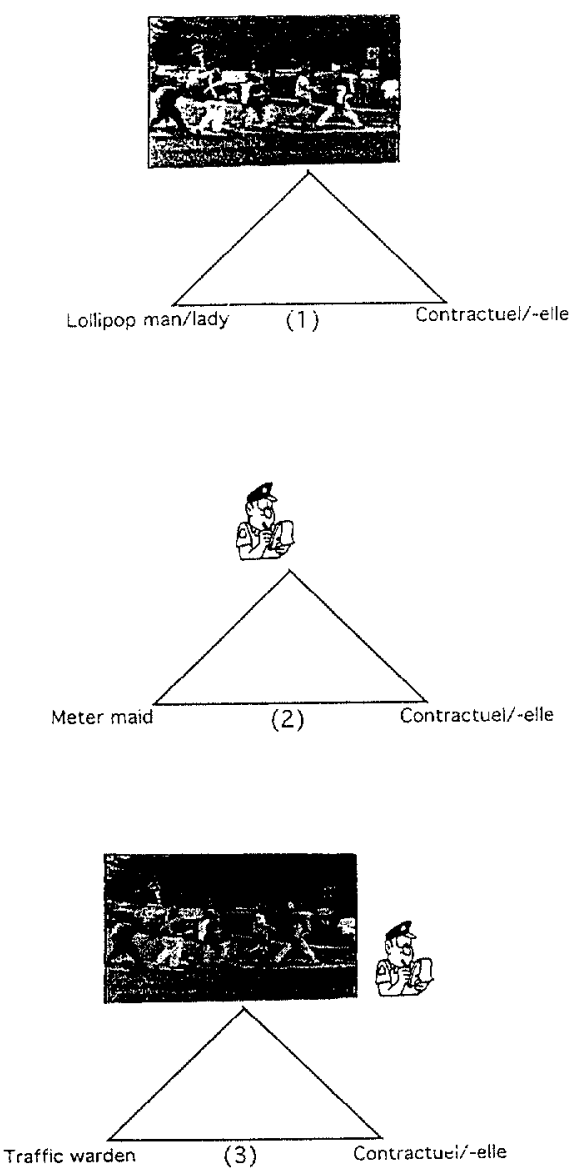
Le premier triangle porte à l'un de ses sommets le terme anglais lollipop man / lady, au second le terme français contractuel/-elle et au troisième le référent des deux termes. Le deuxième triangle porte à un sommet le terme anglais meter maid, à l'autre sommet le terme français contractueli -elle et au troisième sommet le référent. Le troisième triangle comprend à un des sommets le terme anglais traffic warden, à l'autre sommet le terme français contractuel/-elle et au troisième le référent. Dans les trois cas, le référent change. Le terme anglais également. En surface, le terme français reste le même, mais en profondeur il est différent puisqu'il renvoie à des référents différents. Dans le troisième cas, toute l'extension de contractuel/ -elle entre en jeu alors que dans le second, seuls certains de ses caractères spécifiques sont activés, alors que dans le premier cas, ce sont d'autres de ses caractères spécifiques qui sont pris en compte.

Ce n'est que dans le troisième cas, représenté par le triangle dont les sommets sont: traffic warden, contractuel/-elle, référent, qu'on est sûr d'avoir le bon référent. Car comme nous l'avons déjà déclaré, traffic warden et contractuel/-elle ont la même extension et la même compréhension et forcément le même ensemble référentiel. Par contre, dans les deux autres cas, à savoir le triangle dont les sommets sont lollipop man/lady, contractuel/-elle et référent, et le triangle formé par meter maid, contractuel/-elle et référent, il se pose un problème d'ambiguitté sémantique. Qu'on parte du terme anglais (lollipop man/lady, meter maid) vers le terme français (contractuel/ - elle) ou vice versa, le destinataire ou l'interlocuteur ne percevra pas le bon référent. Ce problème est dû au fait que dans le processus traductionnel, on a mis en équivalence un spécifique, ou hyponyme (lollipop man/lady, meter maid) avec un générique ou hyperonyme (contractuel/-elle), soit un élément monosémique avec un élément polysémique. Il faudra mettre un filtre au niveau de l'hyperonyme pour réduire les sèmes de ce dernier. Pour que l'hyperonyme passe à travers ce filtre, il lui faudra un déterminant qui, dans le cas présent peut être stationnement ou enfant. Ainsi aura-t-on contractuel/ -elle de stationnement (meter maid) et contractuel/-elle d' enfants (lollipop man/lady). L'autre solution est celle qu'offre le multimédia en associant l'image (soit le référent) au message:

a) lollipop man/lady - contractuel/-elle - image référentielle d'un(e) contractuel/-elle d'enfants.

b) meter maid - contractuel / -elle - image référentielle d'un(e) contractuelle de stationnement.

\section{c) Extensionnisation ou compréhensionnisation Partie/Tout}

Le problème présenté ci-dessus peut également s'expliquer ou se schématiser dans la relation PARTIE/TOUT (extensionnisation) ou TOUT/PARTIE (compréhensionnisation) comme dans les figure ci-après:

PARTIE / TOUT

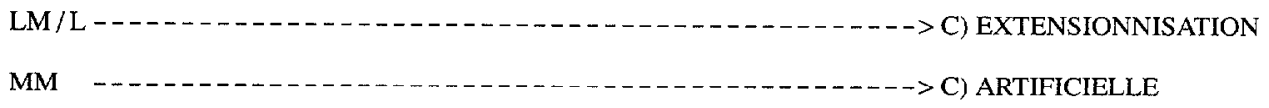

Dans ce schéma, en attribuant à lollipop man/lady et à meter maid (qui ne sont que des sous-ensembles, ou parties du grand ensemble qu'est contractuel/-elle), l'équivalent contractuel/-elle (qui est un ensemble plus grand), on élargit artificiellement le champ sémantique des premiers en leur ajoutant illusoirement des objets qu'en réalité ils n'ont pas. 
Dans ce schéma, en attribuant à contractuel/-elle (qui est un ensemble plus grand) les équivalents lollipop man/lady et meter maid (qui ne sont que des sous-ensembles, donc des parties seulement du grand ensemble qu'est contractuel/-elle), on spécifie artificiellement le champ sémantique du premier en lui ajoutant illusoirement des caractères spécifiques qu'en réalité il n'a pas et en lui ôtant de la même façon des objets qu'en réalité il ne perd pas.

\section{d) Approche topolectale}

Les variantes topolectales sont d'excellentes ressources à exploiter pour résoudre les problèmes d'équivalence extensionnelle ou compréhensionnelle.

Les termes traffic warden, lollipop man / lady et meter maid sont de très bons exemples.

Lollipop man, lollipop lady (et même lollipop woman, expression attestée dans Longman Dictionary of Contemporary English) sont des termes britanniques désignant contractuell-elle d'enfants. Une consultation de la banque de terminologie du Canada (TERMIUM) confirme, par le biais des marques d'usage, le caractère britannique de ces termes, et permet de relever leurs synonymes canadiens, à savoir: crossing guard, crosswalk guard, school patroller, school guard, school crossing guard, safety patroller. À ces termes, TERMIUM donne des équivalents topolectaux appropriés: brigadier, brigadier scolaire, passeur scolaire, passeur. Pour plus de clarté, TERMIUM accompagne ces équivalents de deux définitions, l'une en anglais, l'autre en français :

A person stationed at major highway and street crossings to halt traffic so that children may cross ; usually partially uniformed and carrying a whistle and «stop» sign ; usually employed by the police department on the request of the school board.

Terme (...) pour désigner chez nous la personne qui assure la sécurité des écoliers aux intersections (TERMIUM).

La consultation de quelques dictionnaires confirme les marques d'usage attribuées par TERMIUM. Traffic warden ne figure que dans les dictionnaires britanniques (Collins Cobuild English Language Dictionary de 1988 et Longman Dictionary of Contemporary English de 1987). Il est ainsi défini :

$\mathrm{n}$ BrE an official responsible for controlling the parking of vehicles on city streets (Longman Dictionary of Contemporary English).

A traffic warden is a person whose job is to make sure that cars are not parked where they should not be, and that they are not parked anywhere for longer than is allowed (Collins Cobuild English Language Dictionary).

En conclusion, traffic warden est un terme britannique signifiant contractuel/-elle de stationnement. Alors que meter maid est sa variante topolectale nord-américaine. Meter maid apparait dans la nomenclature du Webster's Ninth New Collegiate Dictionary de 1988 et dans celle du Funk and Wagnalls Canadian College Dictionary de 1986 et est défini de la façon suivante: 
A female member of a police department who is assigned to write tickets for parking violation (Webster's).

A woman employed by a city authority to check parking meters and issue tickets for overtime parking (Funk \& Wagnalls)! .

Meter maid est assez clairement traité dans TERMIUM. Dans cette banque, il a comme synonyme meter checker et comme équivalents préposé(e) au stationnement, auxiliaire de police, auxiliaire au stationnement. TERMIUM donne comme définition et explication à ce terme :

Personne engagée pour faire la surveillance des parcomètres et des infractions aux règlements de stationnement.

L'expression utilisée en France est contractuel(le) : agent de police contractuel qui relève les infractions aux règles de stationnement.

Les termes familiers correspondants sont : souris verte, échalote, bourdon vert (Québec) et aubergine (France) (TERMIUM).

Le terme traffic warden n'est donné qu'à titre indicatif à la fin de la fiche traitant lollipop man. Son équivalent contractuel/-elle n'apparaît presque pas non plus. N'ayant pas eu recours à la notion et aux termes génériques anglais et français qui posent des problèmes d'ordre extensionnel et compréhensionnel, TERMIUM a fait de bons appariements, établissant des liens entre des équivalents absolus qui renvoient à un même référent et qui sont par conséquent facilement exploitables.

Patate - pomme de terre - patate douce

Nous avons trompé la faim en employant l'expression échalote pour parler de préposé(e) au stationnement. Venons-en donc à trois vraies expressions du domaine alimentaire: patate, pomme de terre et patate douce. De quoi parlons-nous réellement? De tubercules comestibles, bien sûr. Toutes les trois expressions renvoient-elles à un même tubercule? La réponse à cette question n'est pas évidente. Elle est à la fois positive et négative. En effet, dans les usages courants, ces expressions entretiennent entre elles des liens de synonymie qu'on peut schématiser de la manière suivante:
A) patate

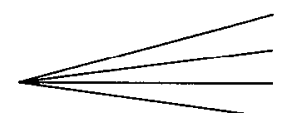
1) pomme de terre
2) pomme
3) patate
4) patate douce
B) pomme de terre

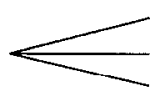
1) pomme de terre
2) pomme
3) patate
C) patate douce
1) patate douce

Dans ces schémas, patate et pomme de terre ont en commun les points 1 (pomme de terre), 2 (pomme) et 3 (patate). Eu égard à ces points, ils sont des synonymes. Patate et patate douce sont également des synonymes; ils ont en commun, quant à eux, les point 4 de patate (patate douce) et, 1 et 2 de patate douce. En d'autres termes, tous les points du schéma B se retrouvent dans le schéma $\mathrm{A}$ et font, par conséquent, de pomme de terre un synonyme de patate. Tous les points du schéma $\mathrm{C}$ se retrouvent dans le schéma $\mathrm{A}$ et font de patate douce un synonyme de patate. Patate est donc une expression polysémique dont - en extension et en compréhension - un des sémèmes est le même que celui de patate douce, à savoir : gros tubercule comestible à chair douceâtre produit par une plante 
rampante vivace à fleurs de liseron, originaire d'Amérique tropicale et qui pousse dans des régions chaudes. L'autre sémème de patate est le même, en extension et en compréhension également, que celui de pomme de terre, soit: tubercule comestible très riche en amidon produit par une plante annuelle herbacée à fleurs blanches, originaire des Andes du Chili et du Pérou. En bref, nous avons : a) patate ${ }_{1}$, synonyme de patate douce, en abrégé patate et b) patate ${ }_{2}$, synonyme de pomme de terre, en abrégé pomme (dans le vocabulaire de la cuisine). Il apparaît évident que patate ${ }_{1}$ et patate $_{2}$ sont deux termes clairement distincts dénommant deux aliments différents.

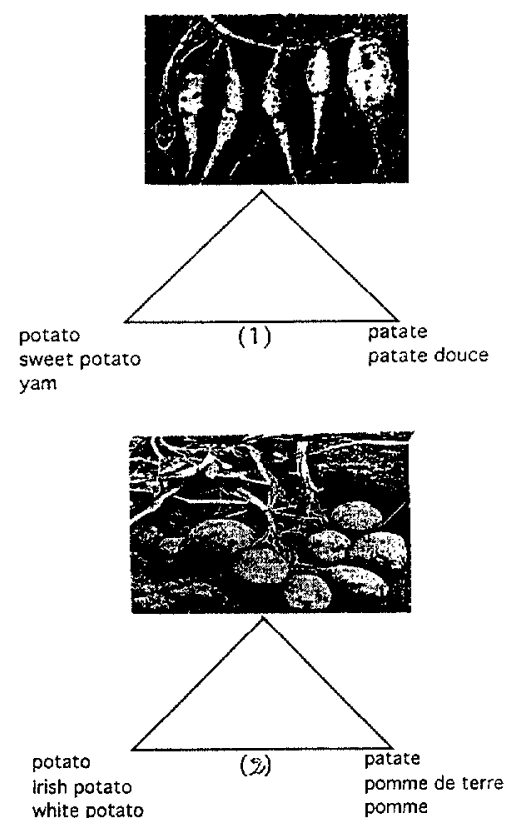

Ces deux termes posent cependant des problèmes d'ordre référentiel, extensionnel et compréhensionnel selon l'aire géographique dans laquelle on en fait usage.

Il se produit, de manière presque générale, une «dé-extensionnisation» de l'expression patate. En effet, sur une même aire, cette expression s'emploie rarement pour les deux extensions patate $_{1}$ et patate $_{2}$. Les locuteurs opèrent un choix entre les deux. Ce faisant, ils «compréhensionnisent» l'expression, en la réduisant aux caractères spécifiques de patate $_{1}$ ou de patate ${ }_{2}$. Dès lors que le choix a été opéré, l'autre extension se voit attribuer plutôt la dénomination synonymique correspondante. À titre d'illustration, soit :

patate:

sémème $1:$ patate $_{1}$, (synonymes) patate douce, patate

sémème 2 : patate 2 , (synonymes) pomme de terre, pomme

Si les locuteurs habitant une aire géographique donnée choisissent d'appeler le sémème1 patate, ils vont dénommer le sémème 2 pomme de terre. S'ils choisissent d'appeler 
plutôt le sémème2 patate, ils vont employer patate douce pour le sémème1. C'est ainsi qu'au Canada et en France, par exemple, on parle de patate (même si cette expression a une connotation familière) pour le sémème 2 et de patate douce pour le sémème 1 . Au Cameroun on emploie patate pour le sémème 1 et pomme de terre pour le sémème2.

Plus haut, nous avons mentionné que pomme de terre a comme synonyme abrégé l'expression pomme. Cette expression engendre une confusion entre le fruit du pommier et la pomme de terre. Pour lever l'ambiguité entre les deux, au Cameroun, les locuteurs marquent la différence en adjoignant un déterminant à pomme (fruit du pommier). Le déterminant adjoint est le nom du pays d'où seraient venues les premières pommes arrivées au Cameroun; il s'agit nommément de la France. Le fruit du pommier avait donc reçu la dénomination pomme de France. Et même si la majorité des pommes qui sont actuellement sur le marché camerounais proviennent d'Afrique du Sud, la mémoire collective a gardé l'appellation initiale.

Quant aux équivalents anglais de patate, ils sont aussi intéressants que les termes français. L'expression potato a également deux sémèmes:

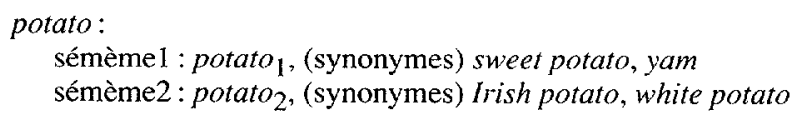

Le sémème $e_{1}$ et le sémème $e_{2}$ de potato sont les équivalents respectifs du sémème ${ }_{1}$ et du sémème ${ }_{2}$ de patate. Dans le sémème ${ }_{1}$, potato est d'usage africain et américain; yam nord-américain alors que sweet potato est nord-américain et britannique. Dans le sémème ${ }_{2}$, potato est d'usage nord-américain et britannique; Irish potato, américain et africain; white potato américain. Ces expressions sont des synonymes topolectaux. La même observation faite pour patate et pomme de terre au sujet de la distribution géographique est valable ici. Une fois que sur une aire géographique, les locuteurs choisissent potato pour un sémème donné, à l'autre sémème ils donnent plutôt la dénomination synonymique de potato. Par exemple, en Afrique, le sémème ${ }_{1}$ est potato et le sémème ${ }_{2}$, Irish potato. En GrandeBretagne, le sémème ${ }_{2} \mathrm{~s}^{\prime}$ appelle potato, et le sémème ${ }_{1}$ est dénommé sweet potato.

Ce principe est suivi même aux États-Unis où existent pourtant toutes les variantes de potato énumérées ci-dessus. Aux États-Unis, il se réalise au niveau régional. Dans le sud, on emploie potato pour le sémème ${ }_{1}$, et pour le sémème ${ }_{2}$ on utilise Irish potato ou white potato. Dans les autres parties du pays, on emploie plutôt les mêmes expressions qu'en Grande-Bretagne, à savoir: potato pour le sémème ${ }_{2}$ et sweet potato pour le sémème ${ }_{1}$.

Aux États-Unis, l'expression yam est utilisée comme synonyme de sweet potato. Il s'agit en fait d'un synonyme extensionnisé, car, tel que le définit Webster's Ninth New Collegiate Dictionary ( «a moist-fleshed and usually orange-fleshed sweet potato»), yam n'est qu'un type de sweet potato. Sans vouloir relancer l'analyse, il est cependant important de préciser qu'en Afrique, cette acception de yam n'est pas connue. Dans ce continent, yam correspond à l'expression scientifique de genus Dioscorea et a pour équivalent français igname et ses extensions (grande igname, igname jaune, igname blanche, igname couscous, igname velue, etc.).

De toutes les expressions que nous venons d'analyser, Irish potato est la seule qui ne figure pas dans les dictionnaires bilingues consultés (Robert \& Collins, Harrap's Shorter, Harrap's Standard). Pour l'établissement des équivalences, nous devons respecter le principe énoncé plus haut. Mais dans un souci d'harmonisation, et les expressions françaises s'y prêtant, nous pouvons recommander que les expressions patate douce et patate soient les équivalents de potato et sweet potato et que pomme de terre et pomme soient ceux de potato, Irish potato et white potato. 


\section{CONCLUSION}

Notre analyse n'a porté que sur quelques langues et expressions. Mais les problèmes qu'elle a révélés sont assez fréquents quand on passe d'une langue à l'autre, ou d'une communauté à l'autre. Les notions, les concepts, voire les objets que nous décrivons ne sont pas toujours identiques. Leurs dénominations non plus. L'extension et la compréhension de ces dénominations encore moins. Même entre les variantes d'une langue, ces problèmes se posent. Entre deux ou plusieurs langues, ils se complexifient. Les traducteurs, les lexicographes bilingues et toutes les personnes que leur travail met en contact avec plusieurs langues ou variantes ont de sérieuses difficultés à cerner les différentes structurations sémantiques, les extensions et les compréhensions de certaines expressions entre lesquelles ils doivent établir des équivalences. Le recours au référent et à des informations spécifiques et topolectales relevant de différents milieux et cultures se révèlent être les meilleurs moyens de désambiguïsation.

Exploitant les ressources de l'autoroute de communication de l'information, la lexicologie informatique câble les chercheurs et leur permet d'accéder aussi bien à la dimension référentielle que topolectale de l'information. Les nouvelles technologies offrent maintenant la possibilité de visualiser le référent, grâce à l'imagerie qui devient de plus en plus présente sur 1'autoroute de communication de l'information et sur multimédia. Elles permettent également l'accès sur réseau, presque partout dans le monde, à des travaux effectués dans différents pays, domaines et langues. Autant les réseaux d'informations sont nombreux, autant l'horizon s'ouvre sur les particularités linguistiques d'ici et d'ailleurs.

\section{Notes}

* Cet article est issu d'une communication présentée par l'auteur aux IVes Journées scientifiques du réseau «Lexicologie, terminologie, traduction» de l'AUPELF-UREF (Lyon, France, 28, 29, 30 septembre 1995).

1. Autrefois, le travail de meter maid était effectué par la gent féminine. Actuellement, il est également fait par les hommes.

\section{RÉFÉRENCES}

A.RNAULD, A, et P. NICOLE (1878) : La logique ou l'art de penser; ouvrage connu sous le nom de Logique de Port-Royal, nouvelle édition publiée avec des arguments, des notes et une table analytique par Émile Charles, Paris, Delagrave, 485 p.

DUVAL, A. (1991) : «L'équivalence dans le dictionnaire bilingue», F. J. Hausmann et al. (dir.), Dictionnaires, encyclopédie internationale de lexicographie, tome troisième, Berlin, Walter de Gruyter, pp. 2817-2824.

GOFFIN, R. (1985) : «La science terminologique», Terminologie et traduction, Bruxelles, Commission des Communautés européennes, $\mathrm{n}^{\circ} 2$, pp. 11-27. 